\title{
Polyphenol oxidase gene family in barley (Hordeum vulgare L.): structural organization and functional activity of the genes in respect to black grain pigment formation
}

\author{
Levanova N.M. ${ }^{1,2 *}$, Glagoleva A.Y. ${ }^{1}$, Khlestkina E.K. ${ }^{1,2,3}$, Shoeva O.Yu. ${ }^{1}$ \\ ${ }^{1}$ Institute of Cytology and Genetics, SB RAS, Novosibirsk, Russia \\ ${ }^{2}$ Novosibirsk State University, Novosibirsk, Russia \\ ${ }^{3}$ N.I. Vavilov All-Russian Institute of Plant Genetic Resources (VIR), St. Petersburg, Russia \\ *e-mail:n.levanova@g.nsu.ru
}

Polyphenol oxidase (PPO) is an enzyme of the class of oxidoreductases. PPO plays an important role in response to abiotic stress and wounding. The enzyme catalase the enzymatic browning reaction in damaged plant tissues by oxidizing $o$-diphenols to highly reactive $o$-quinones. However, its functions in intact tissues have been not well understood. The aim of the current study is to characterize the polyphenol oxidase gene family and to establish their role in formation of the black pigmentation of barley grain. The uncolored cultivar Bowman and its near-isogenic line with black grain i:BwBlp were chosen. Based on the known barley Ppo genes sequences mapped on chromosome $2 \mathrm{H}$ (Ppo1, Ppo2), we identified two more copies: Ppo3 and Ppo4 localized on chromosomes $3 \mathrm{H}$ and $4 \mathrm{H}$, respectively. All copies contain a conservative tyrosinase domain and have an intact tertiary protein structure. Nevertheless, the exon-intron structure of each copy differs as well as their promoter structure. Using the PLACE cis-regulatory elements database, we predicted light-, cold- and drought-responding elements, MYB- and MYCrecognizing elements as well as elements involved in abscisic acid-mediated abiotic stress response (ABRE). We found DRE elements only in the promoters of the Ppo2 and Ppo4 genes and the cold-responding elements only in the Ppo 2 promoter. We analyzed the Ppo gene expression in intact tissues of near-isogenic lines (coleoptile, root, leaf, stem, pericarp and hulls) and in roots and coleoptile of plants exposed to salt stress. We showed that all the Ppo genes in barley have different patterns of expression, while expression of the Ppo2 gene increases in the pericarp and hulls of the $\mathrm{i}: \mathrm{Bw} B l p$ line as the black pigment appears. The Ppo4 gene is not transcriptionally active in any of the intact tissues of both lines.

Acknowledgements: The study was supported by the Russian Science Foundation (No. 16-14-00086). 